\title{
Can Chemical Analysis Predict Wine Aging Capacity?
}

\author{
Andrew L. Waterhouse *(D) and Yingxin Miao \\ Viticulture and Enology, University of California, Davis, CA 95616, USA; yxmiao@ucdavis.edu \\ * Correspondence: alwaterhouse@ucdavis.edu; Tel.: +1-530-752-4777
}

check for

updates

Citation: Waterhouse, A.L.; Miao, Y. Can Chemical Analysis Predict Wine Aging Capacity? Foods 2021, 10, 654. https://doi.org/10.3390/

foods10030654

Academic Editor: Nuno Mateus

Received: 20 January 2021

Accepted: 4 March 2021

Published: 19 March 2021

Publisher's Note: MDPI stays neutral with regard to jurisdictional claims in published maps and institutional affiliations.

Copyright: (c) 2021 by the authors. Licensee MDPI, Basel, Switzerland. This article is an open access article distributed under the terms and conditions of the Creative Commons Attribution (CC BY) license (https:/ / creativecommons.org/licenses/by/ $4.0 /)$.

\begin{abstract}
Oxidation is the limiting factor in wine aging, and recently some famous wines have exhibited unexpected premature oxidation. Antioxidant assays may provide a means to assess a wine's aging potential by measuring its capacity to chemically reduce reagent components. Correlations between antioxidant activity and wine components have the highest value with flavanols, notable for their catechol and phloroglucinol moieties. Both FRAP and DPPH based methods respond strongly to catechol groups, but these functional groups do not protect wine from oxidation. An ideal assay for wine aging capacity would respond selectively to thiols, phloroglucinol moieties, $\mathrm{SO}_{2}$ and other antioxidants capable of reducing quinones. A definitive test will be to compare the various assays against the shelf life of a number of commercial wines.
\end{abstract}

Keywords: oxidation; premox; shelf life; assay

\section{Introduction}

Aging capacity is an essential factor in quality wine. The aging capacity of white Burgundy from the vintage of 1995 to 2000 suddenly dropped, compromising the reputation of older, elegant white Burgundy. The reason was premature oxidation [1]. There were several hypotheses for the reason for premature oxidation, such as less oxygen exposure in the early stages of winemaking, later harvest times, lower sulfur dioxide addition, and corks treated with oxidants. However, all these hypotheses could not fully explain the problem. In addition, the 2003 red Bordeaux showed tired fruit, flabby structure, and were past their prime after ten years of aging. Suggested reasons are the high ripeness of grapes with higher sugar and lower acidity. Lower acidity accelerates the reaction of iron with oxygen, the rate limiting step in oxygen reacting with wine [2]. With global warming, over-ripeness of grapes could become a global issue, leading to a change of grape composition at harvest. The shelf life of some wines could change dramatically. However, the difficult issue is that premature oxidation of a specific new wine is difficult to discern because it usually takes a decade before the problem becomes evident. Thus, accurate prediction of the aging capacity of wine would be very useful.

Wine aging capacity is related to the extent of oxidation with time, where rapid oxidation leads to what is commonly called premox (premature oxidation) in situations where particular wines are expected to age more slowly. The alteration of wine sensory characters due to wine oxidation starts with the degradation of positive aroma, through the development of negative aroma, to browning.

To further understand the wine aging process, the chemical antioxidant compounds in wine were studied. First, oxygen reacts with phenols and yields quinone and hydrogen peroxide. Then, quinones actively react with nucleophiles such as tannin, $\mathrm{SO}_{2}$, amino acid, thiols, and flavan-3-ols [3]. Those nucleophiles are antioxidants with different abilities to react with quinones and aldehydes. This has led to the idea that the shelf life of wine might be related to a wine's antioxidant capacity [4].

The FRAP and DPPH assays are commonly used to determine the antioxidant capacity of samples. In the FRAP assay, an excess of Fe(III), the antioxidants in samples reduce $\mathrm{Fe}(\mathrm{III})$ to form Fe(II) which then forms a complex with TPTZ that can be quantified by 
colorimetric detection at $594 \mathrm{~nm}$. The quantification of $\mathrm{Fe}(\mathrm{II})$ indirectly quantifies the antioxidant capacity of samples [5]. In the DPPH assay, DPPH is a free radical with absorbance at $517 \mathrm{~nm}$. It reacts with antioxidants in the sample, quenching the radical and loss of absorbance indicates the amount of antioxidants in the sample [6]. The reaction between DPPH and radical is shown in Figure 1. Take samples that contain both $\mathrm{SO} 2$ and antioxidants such as catechols as an example, catechols firstly reduce the DPPH and lead to the loss of absorbancc. Then the radical formed from catechol is trapped by DPPH and then slowly release the quionone. Quionone would be reduced the to catechol by sulfite as shown in Figure 2.

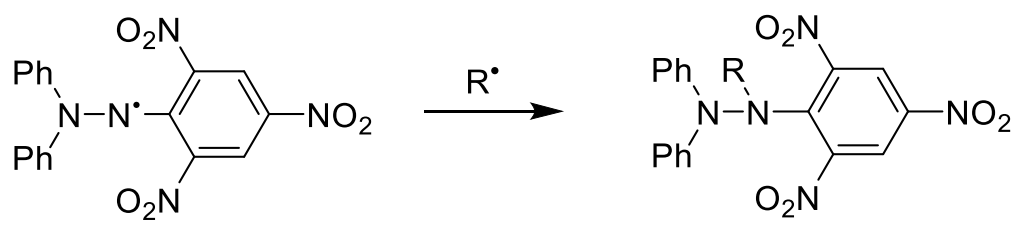

Figure 1. Reaction between DPPH and radical [7].<smiles>[R]C1=CC(=O)C(=O)C(CCc2ccc(O)c([R])c2)=C1</smiles>

Figure 2. The proposed mechanism for the DPPH• oxidation of catechols [8] DPPH.

High-performance liquid chromatography (HPLC) assay and Folin-Ciocalteu (FC) assay are used for phenolic compound analysis. HPLC is capable of quantitative and qualitative analysis of a series of single phenol compounds, S- and N- compounds, such as glutathione (GSH) in wine through the process of extraction, separation, ionization of compounds from samples. FC assay uses the reaction between phenols and the reducible compounds in samples, which produces products with absorption at $765 \mathrm{~nm}$ [8].

Sensory tests are performed to assess the oxidative state of aged wine. Two types of sensory test have been conducted. One method is to score (0-10) on the honey, farm, hay, woody and floral flavors of wine by tasting panels, key descriptors of the oxidation scale. A second method is to score (0-20) on the similarity between samples and an oxidized wine. Comparison between the results of sensory tests and chemical tests could indicate the correlation between chemical changes and sensory changes. The sensory test could inform whether there is a correlation between chemical tests and the formation of oxidized flavors.

This review will explore the possibility of predicting wine aging capacity with existing methods that measure the antioxidant capacity. The mechanisms of these tests will be described to see how they respond to substances known as preservatives. Comparison between the results of those methods, and some tasting results will be presented.

\section{Oxidation and Antioxidant Pathways}

There are two principal oxidants that arise from the primary oxidation reactions, the quinones and aldehydes. The former arise from the oxidation of catechol-type functional groups, and these are common among the phenolics in wine. The quinones are moderately unstable and if there is nothing available, they will decompose over a number of hours in 
solution, and so these are not available to purchase, but for experiments can be made just prior to use. Their preparation is very simple-oxidize the catechol with periodate [9].

In the case of the quinones, antioxidant act by reducing the quinone back to the catechol; some, like ascorbate, do this directly, and some react as nucleophiles which means the catechol is now substituted on one position by the antioxidant. The reaction with sulfite is a good example since both reactions are observed, as shown in Figure 3.<smiles>Cc1ccc(O)c(O)c1</smiles>

Figure 3. The oxidation of 4-methylcatechol by a generic oxidant, followed by reduction with and addition by sulfur dioxide, reversing the process of oxidation.

Other wine nucleophiles that participate in this antioxidation pathway include the A-ring of some flavonoids, due to its phloroglucinol configuration of alternative oxygen atoms, and thiols such as glutathione and cysteine. Other phenolic compounds, for instance caffeic acid, which has only a catechol group, is not a particularly good antioxidant. It is not a good nucleophile, and if it reacted with a quinone, would probably exchange oxidation giving rise to a different quinone, not quenching it [10]. Therefore, an ideal antioxidant test would not be sensitive to the reducing capacity of the catechols, but only to the nucleophilic or quinone reducing power of samples.

In the absence of antioxidants, the quinones will react with other substances, many being flavor molecules, and compromise some desirable flavors. For instance, an important aroma substance, with a citrusy note, is 3-mercaptohexanol (3-MH). This will react quickly with quinones, but so does $\mathrm{SO}_{2}$, and the $\mathrm{SO}_{2}$ scavenges the quinone, preserving the fruity aroma of the 3-MH (Figure 4). Thus for white wines, the fruity flavor can be preserved with some sulfites, while in red wines, the tannin is an additional preservative that supplements any $\mathrm{SO}_{2}$ to also scavenge oxidation and prevent loss of fruity aromas.

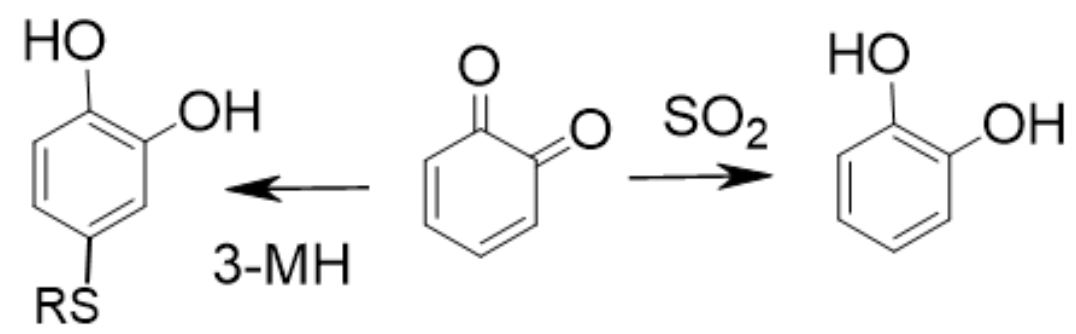

Figure 4. Quinones react quickly with desirable thiols such as 3-MH, but $\mathrm{SO}_{2}$ can prevent the loss of 3-MH by reacting instead, acting as a sacrificial antioxidant.

The second oxidation product, aldehydes, is dominated by acetaldehyde, since its precursor, ethanol, vastly outnumbers other target alcohols. An aroma of oxidation is present when these aldehyde products accumulate in the absence of $\mathrm{SO}_{2}$ [11]. Of the various antioxidants mentioned above, only $\mathrm{SO}_{2}$ can really reduce their formation or hide their presence. These are formed by hydrogen peroxide, the other product of the first step of oxidation, and its reaction with iron to form hydroxyl radical [12]. This radical will oxidize pretty much anything present, but as noted above, the preponderance of ethanol means that most of the oxidation will yield acetaldehyde. $\mathrm{SO}_{2}$ is able to mitigate formation because it will react quickly with hydrogen peroxide [13], avoiding the radical formation. Of the other antioxidants, glutathione and ascorbate will react with hydrogen peroxide [14], though usually ascorbate is not present at significant levels unless added. 
However, then, even when some acetaldehyde is formed, the $\mathrm{SO}_{2}$ will bind it to make the sulfonate, rendering it and related aldehydes non-volatile. Glutathione and phloroglucinol will also react with acetaldehyde, but only partially, reducing, but not eliminating oxidation aromas [15].

\section{Oxidation Tests}

The basis of the FRAP assay is the conversion of iron (III) to iron (II) a one electron reduction step. The iron (II) is then visualized by complexation with ferrozine, and levels down to $2 \mu \mathrm{M}$ ascorbic acid equivalent $(350 \mu \mathrm{g} / \mathrm{L}$ ) can be observed [5]. In wine, the primary reducing species is the catechol functional group, which is converted to the quinone form via a semi-quinone radical. The catechol functional group is abundant on condensed tannin found in red wine, as well in the hydroxycinnamates found in both red and white wine. On a mass or molar basis, such phenolics are by far the most abundant substance in wine that can reduce iron (III). However, other reducing species could also participate in this reduction step, and ascorbic acid is very effective at reducing iron, while the thiol glutathione can reduce iron, but is much weaker than catechols [16]. Sulfite is potent in the FRAP assay [8]. Mono-hydroxy phenols are also fairly ineffective at this reduction, generally at least two orders of magnitude lower, as the monohydroxy compounds do not easily oxidize to the quinone form, and meta-dihydroxy compounds are also weak for the same reason. [17].

However, aside from a direct reaction with iron (III), some antioxidants are good nucleophiles that can react with the immediate oxidation product, the quinones, very quickly yielding a catechol, and reversing the oxidation process. After this reversal, the newly formed catechol can react again, so even nucleophiles that do not react with iron (III) can enhance its reduction almost stoichiometrically. These include the thiols, sulfite and the phloroglucinol functional group found on many flavonoids [18], as well as amino acids [19]. The effectiveness of these various compounds in enhancing the response in an FRAP test will depend on the rate of their reaction with quinones. In other words, will they react in the time frame of the assay? In some cases, this is not well known.

Therefore, these substances should be able to enhance the response of antioxidant tests, even if they do not have a direct response. For instance while glutathione has a weak response in the FRAP assay (0.03), it greatly enhances the response of caffeic acid (1.76). Half doses of each of these two substances combined would be expected to give a response of 0.9 if their combination was additive, but that combination actually gave a response of 2.21, showing how effective glutathione is at reducing quinone back to the catechol [20]. Other have observed a similarly enhanced synergy between a catechol-type compound and glutathione [21].

A similar effect can be observed with different antioxidant tests, but the magnitude of the synergy will vary depending on the capacity of the additive to react directly with the colorimetric reaction [21]. In the Sun example, glutathione has fairly good capacity to quench radicals, so in the DPPH assay, it has a nearly additive effect with caffeic acid. Additionally, ascorbic acid is very effective at reducing iron (III), and so its effect is very nearly additive when combined with other good substrates in the FRAP assay, Figure 5.

In attempting to measure the capacity of a wine to "absorb" oxygen, it is important to distinguish between phenolic substances which are the substrates of oxidation and lead to reactive oxidation intermediates, such as quinones, and those substances which can effectively reverse the oxidation process by quenching those quinones before they can react with flavor molecules. Sulfur dioxide is excellent at this task, as is ascorbate and glutathione. A recent report showed that using LC-MS, it was possible to quantify the amount of substances that would react with quinones by adding a model quinone and looking for all the representative products, the oxidation metabolome [19]. While this provided excellent insight into the inventory of antioxidants, this complex measurement is not practical for production analysis. 


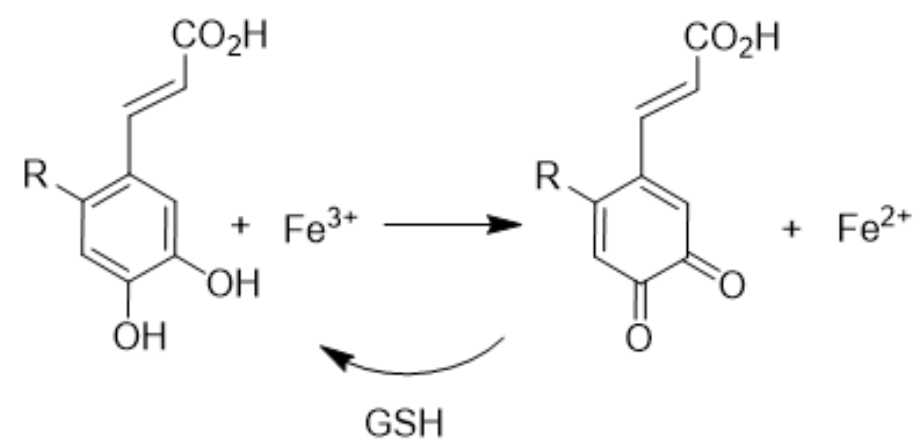

Figure 5. Production of iron (II), the colorimetric product of the FRAP assay, by caffeic acid, and the recycling of the quinone product back to the reactant by glutathione (GSH). This recycling will result in producing more iron (II) per unit of caffeic acid. R=H or GS (glutathionyl).

\section{Comparison among Results from FRAP, DPPH, HPLC and FC Assay}

Ten high quality, single variety red wine samples vintage 1998 made in Greece were tested after three-years aging. The results in Table 1 of wine made from various grape varieties were significantly different regardless of the identical aging period. It was a good sampling because the range of results on the chemical assays was nearly a factor of two to a factor of 4 for the anthocyanin amounts, and that provided a fairly good range for comparisons to the antioxidant tests seen in Table 2.

Table 1. Polyphenolic Composition and Antioxidant Properties of the Wines Examined [22].

\begin{tabular}{ccccccc}
\hline Wine Code & $\begin{array}{c}\text { Total } \\
\text { Phenolics }\end{array}$ & $\begin{array}{c}\text { Total } \\
\text { Flavanols }\end{array}$ & $\begin{array}{c}\text { Total } \\
\text { Anthocyanins }\end{array}$ & A AR $_{\text {AR }}$ & SA $_{\text {HFR }}$ & P \\
\hline 1 & $2117 \pm 117$ & $608.1 \pm 3.5$ & $101.8 \pm 16.5$ & $1.46 \pm 0.07$ & $54.7 \pm 0.4$ & $11.03 \pm 0.15$ \\
2 & $2276 \pm 323$ & $606.5 \pm 2.7$ & $81.1 \pm 2.5$ & $1.48 \pm 0.06$ & $58.0 \pm 1.1$ & $10.27 \pm 0.21$ \\
3 & $3098 \pm 112$ & $473.0 \pm 6.6$ & $212.6 \pm 2.0$ & $1.15 \pm 0.07$ & $47.8 \pm 1.3$ & $8.64 \pm 0.05$ \\
4 & $3450 \pm 171$ & $568.5 \pm 1.2$ & $248.4 \pm 2.1$ & $1.32 \pm 0.07$ & $53.0 \pm 0.9$ & $9.06 \pm 0.07$ \\
5 & $2898 \pm 186$ & $596.7 \pm 0.7$ & $363.1 \pm 2.4$ & $1.42 \pm 0.08$ & $60.0 \pm 1.9$ & $10.59 \pm 0.05$ \\
6 & $1757 \pm 145$ & $347.0 \pm 10.9$ & $136.7 \pm 5.0$ & $1.10 \pm 0.02$ & $43.7 \pm 1.5$ & $5.86 \pm 0.07$ \\
7 & $1217 \pm 292$ & $339.1 \pm 6.1$ & $109.7 \pm 20.1$ & $0.89 \pm 0.06$ & $42.2 \pm 0.9$ & $5.36 \pm 0.04$ \\
8 & $1328 \pm 235$ & $424.4 \pm 7.4$ & $271.2 \pm 18.5$ & $0.88 \pm 0.06$ & $46.8 \pm 0.6$ & $5.49 \pm 0.09$ \\
9 & $3772 \pm 284$ & $664.8 \pm 9.5$ & $360.1 \pm 9.9$ & $1.53 \pm 0.07$ & $61.4 \pm 0.5$ & $10.80 \pm 0.18$ \\
10 & $3287 \pm 250$ & $643.6 \pm 6.0$ & $121.7 \pm 3.8$ & $1.39 \pm 0.04$ & $57.0 \pm 1.0$ & $8.35 \pm 0.14$ \\
ave & 2390 & 527 & 201 & 1.26 & 52.5 & 8.55 \\
\hline
\end{tabular}

Total phenolics (mg/L gallic acid) by F-C; $\mathrm{A}_{\mathrm{AR}}$ (mM Trolox) by DPPH assay; $\mathrm{P}_{\mathrm{R}}$ (mM ascorbic acid) by FRAP assay, total flavanols (mg L-1 catechin) by DMACA method.

Table 2. Correlations between DPPH, FRAP assay and total phenolics (TP), total flavanols (TF) and total anthocyanins (TA) concentration. [22].

\begin{tabular}{cc}
\hline Values Correlated & Corresponding Correlation Coefficients $\left(\mathbf{r}^{\mathbf{2}}\right)$ \\
\hline DPPH-TP & 0.523 \\
FRAP-TP & 0.465 \\
DPPH-TF & 0.842 \\
FRAP-TF & 0.786 \\
DPPH-TA & 0.018 \\
FRAP-TA & 0.060 \\
\hline
\end{tabular}

In Table 2, the results from FRAP and DPPH showed relatively low correlation with the results from the FC assay (Total phenolics) and total anthocyanins concentration. The results from FRAP and DPPH assay showed relatively high correlation with total flavanols. This suggests that the flavanols in wine are strong contributors to the antioxidant ability of wine. 
This correlation is not surprising since the flavanols all contain both a catechol (or galloyl) functional group that can reduce iron (III), or donate a hydrogen to the DPPH radical. In addition, the flavanols contain a phloroglucinol group on the A-ring, which can act as a nucleophile and reduce any oxidized catechol quinone back to the dihydro form by addition, enhancing the antioxidant effect. The correlation with phenolics would be expected to be smaller because some phenolics lack a catechol group, such as coumaric acid or isorhamnetin, and thus cannot reduce iron (III) and the anthocyanins are dominated by malvidin, which lacks a catechol group. Wine also contains other antioxidant substances, such as glutathione, not measured here, but if present, can add noise to a correlation between phenolic assays and antioxidant assays by enhancing the result.

In another study from the same research team, 25 aged red wine (vintage 1998, aged for 7 years) [23] were tested by F-C assay, FRAP assay and DPPH assay as shown in Table 3. The total phenol levels ranged from 1200 to $>3700$, a range of over three, while the total flavanol levels went from 214 to 922, a more substantial range than the prior study.

Table 3. Polyphenolic composition and antioxidant parameters of the wines tested $(n=3)$ [23].

\begin{tabular}{ccccc}
\hline Wine & TP & TF & $\mathbf{A}_{\mathbf{A R}}$ & $\mathbf{P}_{\mathbf{R}}$ \\
\hline Archanes & $3061 \pm 211$ & $665.3 \pm 11.8$ & $0.98 \pm 0.04$ & $8.13 \pm 0.12$ \\
Archanes & $1543 \pm 218$ & $302.6 \pm 9.5$ & $0.79 \pm 0.02$ & $5.80 \pm 0.09$ \\
Goumenissa & $2613 \pm 150$ & $546.8 \pm 4.4$ & $1.13 \pm 0.07$ & $9.04 \pm 0.10$ \\
Goumenissa & $2165 \pm 189$ & $214.2 \pm 6.1$ & $1.08 \pm 0.09$ & $6.49 \pm 0.06$ \\
Naoussa & $2117 \pm 117$ & $608.1 \pm 3.5$ & $1.46 \pm 0.07$ & $11.03 \pm 0.15$ \\
Naoussa & $2276 \pm 323$ & $606.5 \pm 2.7$ & $1.48 \pm 0.06$ & $10.27 \pm 0.21$ \\
Nemea & $3098 \pm 112$ & $473.0 \pm 6.6$ & $1.15 \pm 0.07$ & $8.64 \pm 0.05$ \\
Nemea & $3450 \pm 171$ & $568.5 \pm 1.2$ & $1.32 \pm 0.07$ & $9.06 \pm 0.07$ \\
Nemea & $2898 \pm 186$ & $596.7 \pm 0.7$ & $1.42 \pm 0.08$ & $10.59 \pm 0.05$ \\
Paros & $3606 \pm 51$ & $922.3 \pm 3.2$ & $1.42 \pm 0.06$ & $11.54 \pm 0.24$ \\
Kritikos Topikos & $1939 \pm 96$ & $291.1 \pm 10.4$ & $0.77 \pm 0.00$ & $5.29 \pm 0.04$ \\
Kritikos Topikos & $1658 \pm 199$ & $309.7 \pm 3.1$ & $0.85 \pm 0.01$ & $6.51 \pm 0.05$ \\
Kritikos Topikos & $1709 \pm 34$ & $444.7 \pm 11.2$ & $0.97 \pm 0.02$ & $8.14 \pm 0.10$ \\
Macedonikos Topikos & $1217 \pm 292$ & $339.1 \pm 6.1$ & $0.73 \pm 0.02$ & $5.36 \pm 0.04$ \\
Peloponissiakos Topikos & $2091 \pm 57$ & $442.9 \pm 3.8$ & $0.87 \pm 0.01$ & $7.11 \pm 0.07$ \\
Peloponissiakos Topikos & $1780 \pm 181$ & $346.3 \pm 11.2$ & $1.04 \pm 0.03$ & $8.18 \pm 0.22$ \\
Topikos Dramas & $2439 \pm 278$ & $650.4 \pm 19.6$ & $1.14 \pm 0.05$ & $8.57 \pm 0.11$ \\
Topikos Chalkidikis & $1328 \pm 235$ & $424.4 \pm 7.4$ & $0.88 \pm 0.01$ & $5.49 \pm 0.09$ \\
Topikos Florinas 19 & $2187 \pm 231$ & $546.8 \pm 7.3$ & $1.08 \pm 0.02$ & $7.83 \pm 0.06$ \\
Topikos Imathias & $3772 \pm 284$ & $664.8 \pm 9.5$ & $1.37 \pm 0.05$ & $10.80 \pm 0.18$ \\
Topikos Letrinon & $2354 \pm 223$ & $352.2 \pm 2.4$ & $0.91 \pm 0.05$ & $6.63 \pm 0.01$ \\
Topikos Op. Lokridos & $2243 \pm 245$ & $435.9 \pm 3.0$ & $1.03 \pm 0.02$ & $7.39 \pm 0.02$ \\
Topikos Plagionn Egialias & $3287 \pm 351$ & $643.6 \pm 6.0$ & $1.22 \pm 0.01$ & $8.35 \pm 0.14$ \\
Topikos Stereas Elladas & $1943 \pm 274$ & $380.2 \pm 10.8$ & $0.94 \pm 0.02$ & $6.23 \pm 0.01$ \\
Topikos Tegeas & $1995 \pm 256$ & $383.0 \pm 10.1$ & $0.99 \pm 0.02$ & $7.12 \pm 0.02$ \\
Average & 2351 & 526.8 & 1.05 & 8.02 \\
\hline
\end{tabular}

TP: Total phenolics (mg/L gallic acid) by F-C; TF: Total flavanols (mg/L catechin); $\mathrm{A}_{\mathrm{AR}}$ (mM Trolox) by DPPH assay; $\mathrm{P}_{\mathrm{R}}(\mathrm{mM}$ ascorbic acid) by FRAP assay.

As shown in Table 4, the correlation coefficient between total flavanols and FRAP value $\left(r^{2}=0.6850\right)$ was again higher than that of between total phenols and the FRAP value $\left(\mathrm{r}^{2}=0.3884\right)$, although the TF-FRAP correlation was weaker than in the earlier study. However, these results still indicate that the flavanols play a major role in the chemical antioxidant capacity of wine, a key factor in the aging of red wine, where the bulk of the phenolic compounds are condensed tannin, made of flavanol units. 
Table 4. Statistical parameters as calculated from correlations established using regression analysis at a $99.9 \%(p=0.001)$ [23].

\begin{tabular}{cc}
\hline Values Correlated & Corresponding Correlation Coefficients $\left(\mathbf{r}^{\mathbf{2}}\right)$ \\
\hline DPPH-TP & 0.4420 \\
FRAP-TP & 0.3884 \\
DPPH-TF & 0.5508 \\
FRAP-TF & 0.6850 \\
DPPH-TA & 0.1168 \\
FRAP-TA & 0.1006 \\
\hline
\end{tabular}

TP (Total phenolics) (mg/L gallic acid) by F-C; AAR (mM Trolox) by DPPH assay; PR (mM ascorbic acid) by FRAP assay, TF (total flavanols).

In a study of the response of white wines to antioxidant tests, the sulfur dioxide in four white wines were largely removed by adding an aqueous hydrogen peroxide solution [8]. The treated samples were compared with the original wines as shown in Table 5. The treated samples with no free $\mathrm{SO}_{2}$ showed much lower FRAP value than original samples. However, DPPH assay and FC assay showed relatively smaller differences between the $\mathrm{SO}_{2}$-removed sample and original samples. This suggested that the DPPH value and F-C test were less affected by $\mathrm{SO}_{2}$ in samples. After $\mathrm{SO}_{2}$ removal, the measurements of $\mathrm{DPPH}$, FRAP, F-C total phenol assay ranked the wine samples similarly. The Sauvignon blanc from South Africa and France showed the highest results, then the Soave from Italy and the Pinot grigio showed the lowest value.

Table 5. Comparison of results obtained with original white wines and after $\mathrm{SO}_{2}$ was largely removed [8].

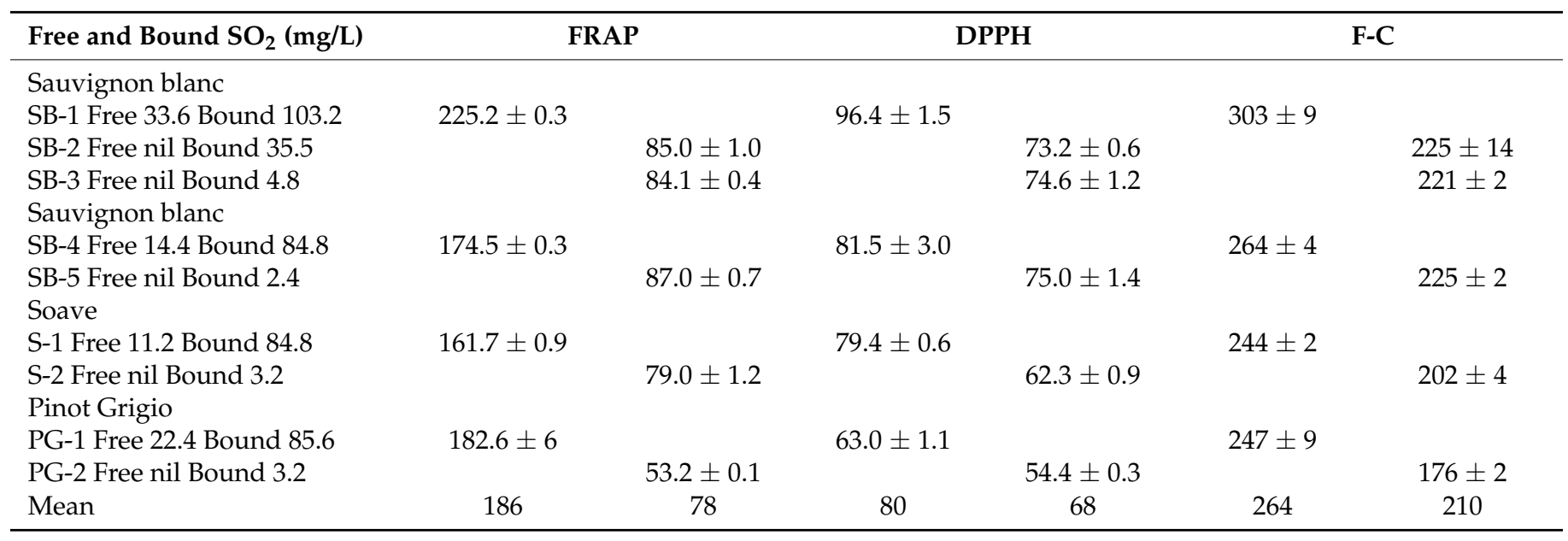

FRAP and DPPH (mg/L Caffeic acid equivalent), F-C (mg/L gallic acid equivalent). Caffeic acid equivalent $=($ E320*1.4) $\times 11.1(\mathrm{mg} / \mathrm{L})$ [24].

\subsection{The Age of Wine and Antioxidant Activity}

A study of California wines of different ages compared age with various phenolic and antioxidant parameters [25], see Table 6. The wine samples were red wines of 1-28 years old. With increasing wine age, the concentration of monomeric phenolics and LMWP decreased, while the concentration of HMWP increased. However, there was no significant correlation between the wine age and total phenols or antioxidant capacity. The values varied unpredictably with wine age. This study did not look at chemically distinct categories of phenolic compounds, so the phenolic factors that might be affecting these differences could not be assessed. 
Table 6. Levels of wine phenolic monomers, low molecular-weight polymers (LMWP) and high molecular-weight polymers (HMWP) as determined by using HLPC and parameters characterizing AOA given by Folin-Ciocalteau total phenols, CV response (Q500) and overall AOA (N) See [25] for details.

\begin{tabular}{|c|c|c|c|c|c|c|}
\hline $\begin{array}{l}\text { Grape and } \\
\text { Vintage }\end{array}$ & $\begin{array}{c}\text { Monomers } \\
\text { (\% Area) }\end{array}$ & $\begin{array}{l}\text { LMWP } \\
\text { (\% Area) }\end{array}$ & $\begin{array}{l}\text { HMWP } \\
\text { (\% Area) }\end{array}$ & $\begin{array}{c}\text { FC } \\
(\mathrm{mg} / \mathrm{L} \text { GAE) }\end{array}$ & $\begin{array}{c}\mathrm{Q}_{500} \\
\text { (mg/L CE) }\end{array}$ & $\mathbf{N}(\mathbf{m M})$ \\
\hline Cabernet 1999 & 20.3 & 13 & 66.7 & 1193 & 1655 & 12.8 \\
\hline Cabernet 1999 & 17.3 & 12.7 & 70 & 2361 & 3157 & 27.9 \\
\hline Cabernet 1998 & 24 & 13.6 & 62.4 & 1162 & 1366 & 12 \\
\hline Cabernet 1984 & 16 & 8.6 & 75.4 & 1897 & 1493 & 20.6 \\
\hline Cabernet 1977 & 9.8 & 8.2 & 82 & 2379 & 2099 & 27.9 \\
\hline Cabernet 1973 & 17.5 & 9.9 & 72.6 & 1002 & 941 & 11.6 \\
\hline Zinfandel 1999 & 20.3 & 15.2 & 64.5 & 2152 & 2372 & 24.4 \\
\hline Zinfandel 1999 & 24.2 & 16.7 & 59.1 & 2457 & 3336 & 24.7 \\
\hline Zinfandel 1994 & 29.4 & 19.1 & 51.5 & 689 & 752 & 7.9 \\
\hline Zinfandel 1989 & 18.6 & 10.6 & 70.8 & 1854 & 2011 & 19.8 \\
\hline Pinot Noir 2000 & 35.2 & 18.2 & 46.6 & 1711 & 2504 & 17.8 \\
\hline Pinot Noir 1999 & 28.5 & 18 & 53.5 & 1693 & 1890 & 20.1 \\
\hline Pinot Noir 1998 & 27 & 17 & 56 & 1563 & 1180 & 17.8 \\
\hline Pinot Noir 1992 & 27.7 & 16.7 & 55.6 & 930 & 1573 & 15.6 \\
\hline Pinot Noir 1984 & 20.5 & 10.1 & 69.4 & 1655 & 1941 & 20.4 \\
\hline Merlot 1999 & 19.9 & 16.4 & 63.7 & 2410 & 4819 & 29.3 \\
\hline Merlot 1999 & 19.3 & 14.7 & 66 & 2616 & 3949 & 28.2 \\
\hline Barbera 2001 & 35.1 & 19 & 45.9 & 1597 & 1335 & 17.1 \\
\hline Barbera 1999 & 34.6 & 21 & 44.4 & 1155 & 1672 & 15.6 \\
\hline Grenach 2001 & 27.3 & 17.3 & 55.4 & 996 & 1738 & 18.6 \\
\hline Carmine 1986 & 17.8 & 10.7 & 71.5 & 980 & 1165 & 12 \\
\hline
\end{tabular}

Another study looking at antioxidant capacity versus vintage age had a different result [26], see Figure 6. The wine samples are white wine of nine vintages from the same vineyard. With increasing wine age, the antioxidant capacity of wine consistently decreased until the wine was about 10 years old. The difference in the results of these two studies is likely due to the fact that the second project had good control over many factors which might influence the wines' composition because all the grapes were from the same vineyard.

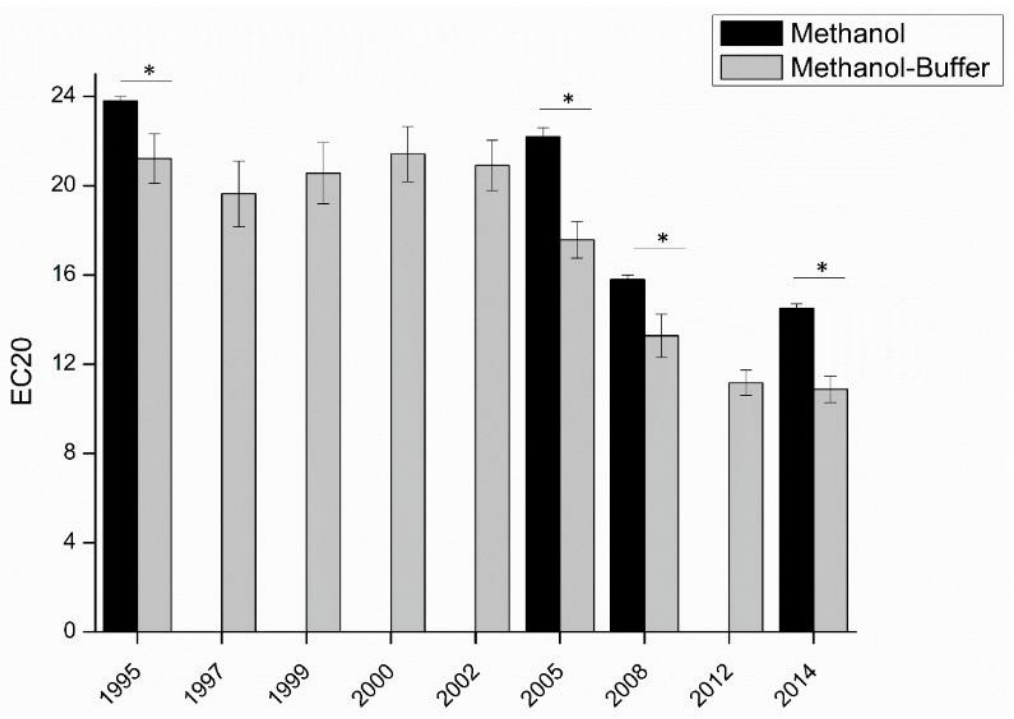

Figure 6. Comparison of the antioxidant capacity of the same appellation from nine vintages from the same winery. Measurements in methanol (black), and in methanol-buffer (grey). Student tests show comparison between methanol and methanol-buffer methods, "**" indicate significant difference $(p<0.05)$. [26]. 


\subsection{Antioxidant Analysis vs. Sensory Tests}

In a diagnostic experiment, wine samples were measured for antioxidant content by a titration, and that result was compared to oxidized character in the wine after forced aging [27]. In Table 7, Group I was the forced aged wine and in Group II were wine of 1-20 years old. The coefficients of correlation between the sensorial (ID) and ROX results from potentiometric titration assay were high for both forced aging wine samples and the age of the group II wine samples. It would appear that the redox titration was a good predictor of resistance to oxidation, albeit under forcing conditions. It would be important to know if this measurement could predict the appearance of oxidized character of wine under normal aging.

Table 7. Coefficients of correlation between sensorial descriptors and dROX Values from Group I and Group II [27].

\begin{tabular}{ccc}
\hline Corr Coeff (r) with ROX & $\begin{array}{c}\text { Wine Group I Forced Aging } \\
\text { Experiment }(\boldsymbol{n = 1 3 )}\end{array}$ & $\begin{array}{c}\text { Wine Group II Commercial } \\
\text { Wines }(\boldsymbol{n}=\mathbf{2 4})\end{array}$ \\
\hline ID & 0.8869 & 0.8725 \\
floral & -0.9068 & -0.7728 \\
honey-like & 0.8815 & 0.7826 \\
hay & 0.9465 & 0.8252 \\
woody-like & 0.9358 & 0.8411 \\
farm feed & 0.8628 & 0.8286 \\
\hline
\end{tabular}

\section{Conclusions}

A wine's aging ability is limited by its oxidation, but can antioxidant assays predict a wine's aging potential? The various options, such as FRAP, DPPH, voltammetry, and potentiometric titration respond to phenolic compounds as well as protective antioxidants such as $\mathrm{SO}_{2}$. Thus, the results of wine antioxidant ability and chemical analysis of polyphenol compounds are correlated, especially for flavanols. Some potentiometric tests showed a good correlation with resistance to oxidation in accelerated aging.

Future work involves testing simple catechols and non-catechol antioxidants with candidate tests, and then with the mixtures to see which can act to synergize their activity in the test beyond the additive effect, by recycling the oxidized form back to the catechol form. Even better would be a test that does not measure catechols, but all the substances that can react with quinones and reverse the oxidation process. The reason for that focus is that the recycling effect is the key to avoiding oxidation, while the catechols are not a strong factor in preventing wine oxidation, but in fact are the pathway to oxidation. A LC-MS assay has recently been shown to document these factors, but the nature of a routine test that could reveal the same is not obvious. A definitive test will be to compare the various assays against the shelf life of a number of commercial wines.

Author Contributions: Conceptualization, A.L.W.; Y.M.; writing-original draft preparation, Y.M.; writing-review and editing, A.L.W. All authors have read and agreed to the published version of the manuscript.

Funding: This research received no external funding.

Conflicts of Interest: The authors declare no conflict of interest.

\section{References}

1. Colman, T. The Puzzling Problem of Premox. Features. Wine Searcher. 2013. Available online: https://www.wine-searcher.com/ $\mathrm{m} / 2013 / 09 /$ the-puzzling-problem-of-premox (accessed on 19 January 2021).

2. Nguyen, T.H.; Waterhouse, A.L. Redox Cycling of Iron: Effects of Chemical Composition on Reaction Rates with Phenols and Oxygen in Model Wine. Am. J. Enol. Vitic. 2021, 73. [CrossRef]

3. Waterhouse, A.L.; Sacks, G.L.; Jeffery, D.W. Understanding Wine Chemistry; John Wiley \& Sons, Ltd.: Chichester, West Sussex, UK, 2016; p. 443. 
4. Escudero, A.; Asensio, E.; Cacho, J.; Ferreira, V. Sensory and chemical changes of young white wines stored under oxygen. An assessment of the role played by aldehydes and some other important odorants. Food Chem. 2002, 77, 325-331. [CrossRef]

5. Benzie, I.F.F.; Strain, J.J. Ferric reducing antioxidant power assay: Direct measure of total antioxidant activity of biological fluids and modified version for simultaneous measurement of total antioxidant power and ascorbic acid concentration. Methods Enzymol. 1999, 299, 15-27. [PubMed]

6. Sharma, O.P.; Bhat, T.K. DPPH antioxidant assay revisited. Food Chem. 2009, 113, 1202-1205. [CrossRef]

7. Abuin, E.; Lissi, E.; Ortiz, P.; Henriquez, C. Uric acid reaction with DPPH radicals at the micellar interface. Bol. Soc. Chil. Quim. 2002, 47, 145-149. [CrossRef]

8. Danilewicz, J.C. Folin-Ciocalteu, FRAP, and DPPH• Assays for Measuring Polyphenol Concentration in White Wine. Am. J. Enol. Vitic. 2015, 66, 463-471. [CrossRef]

9. Gislason, N.E.; Currie, B.L.; Waterhouse, A.L. Novel Antioxidant Reactions of Cinnamates in Wine. J. Agric. Food Chem. 2011, 59, 6221-6226. [CrossRef]

10. Ma, L.J.; Waterhouse, A.L. Flavanols react preferentially with quinones through an electron transfer reaction, stimulating rather than preventing wine browning. Anal. Chim. Acta 2018, 1039, 162-171. [CrossRef]

11. Cullere, L.; Ferreira, V.; Cacho, J. Analysis, occurrence and potential sensory significance of aliphatic aldehydes in white wines. Food Chem. 2011, 127, 1397-1403. [CrossRef]

12. Moffett, J.W.; Zika, R.G. Reaction-kinetics of hydrogen-peroxide with copper and iron in seawater. Environ. Sci. Technol. 1987, 21, 804-810. [CrossRef]

13. McArdle, J.V.; Hoffmann, M.R. Kinetics and mechanism of the oxidation of aquated sulfur-dioxide by hydrogen-peroxide at low pH. J. Phys. Chem. 1983, 87, 5425-5429. [CrossRef]

14. Hasan, S.M.K.; Scampicchio, M.; Ferrentino, G.; Kongi, M.O.; Hansen, L.D. Thermodynamics and kinetics of the Fenton reaction in foods. Thermochim. Acta 2019, 682, 8. [CrossRef]

15. Peterson, A.L.; Waterhouse, A.L. H-1 NMR: A Novel Approach To Determining the Thermodynamic Properties of Acetaldehyde Condensation Reactions with Glycerol, (+)-Catechin, and Glutathione in Model Wine. J. Agric. Food Chem. 2016, 64, 6869-6878. [CrossRef] [PubMed]

16. Berker, K.I.; Guclu, K.; Demirata, B.; Apak, R. A novel antioxidant assay of ferric reducing capacity measurement using ferrozine as the colour forming complexation reagent. Anal. Methods 2010, 2, 1770-1778. [CrossRef]

17. Spiegel, M.; Kapusta, K.; Kolodziejczyk, W.; Saloni, J.; Zbikowska, B.; Hill, G.A.; Sroka, Z. Antioxidant Activity of Selected Phenolic Acids-Ferric Reducing Antioxidant Power Assay and QSAR Analysis of the Structural Features. Molecules 2020, 25, 3088. [CrossRef] [PubMed]

18. Nikolantonaki, M.; Waterhouse, A.L. A Method to Quantify Quinone Reaction Rates with Wine Relevant Nucleophiles: A Key to the Understanding of Oxidative Loss of Varietal Thiols. J. Agric. Food Chem. 2012, 60, 8484-8491. [CrossRef] [PubMed]

19. Romanet, R.; Bahut, F.; Nikolantonaki, M.; Gougeon, R.D. Molecular Characterization of White Wines Antioxidant Metabolome by Ultra High Performance Liquid Chromatography High-Resolution Mass Spectrometry. Antioxidants 2020, 9, 115. [CrossRef] [PubMed]

20. Capitani, C.D.; Carvalho, A.C.L.; Botelho, P.B.; Carrapeiro, M.M.; Castro, I.A. Synergism on antioxidant activity between natural compounds optimized by response surface methodology. Eur. J. Lipid Sci. Technol. 2009, 111, 1100-1110. [CrossRef]

21. Sun, Y.; Yu, Y.Y.; Cao, S.W. Antioxidant Capacity of Caffeic Acid, Phloretin and Glutathione Mixtures and Formula Optimization. Asian J. Chem. 2013, 25, 3971-3978. [CrossRef]

22. Arnous, A.; Makris, D.P.; Kefalas, P. Effect of principal polyphenolic components in relation to antioxidant characteristics of aged red wines. J. Agric. Food Chem. 2001, 49, 5736-5742. [CrossRef]

23. Makris, D.P.; Kefalas, P. Association between in vitro antiradical activity and ferric reducing power in aged red wines: A mechanistic approach. Food Sci. Technol. Int. 2005, 11, 11-18. [CrossRef]

24. Somers, T.C.; Ziemelis, G. Spectral Evaluation of Total Phenolic Components in Vitis vinifera: Grapes and Wine. J. Sci. Food Agric. 1985, 36, 1275-1284. [CrossRef]

25. Roginsky, V.; de Beer, D.; Harbertson, J.F.; Kilmartin, P.A.; Barsukoval, T.; Adams, D.O. The antioxidant activity of Californian red wines does not correlate with wine age. J. Sci. Food Agric. 2006, 86, 834-840. [CrossRef]

26. Romanet, R.; Coelho, C.; Liu, Y.Z.; Bahut, F.; Ballester, J.; Nikolantonaki, M.; Gougeon, R.D. The Antioxidant Potential of White Wines Relies on the Chemistry of Sulfur-Containing Compounds: An Optimized DPPH Assay. Molecules 2019, 24, 1353. [CrossRef]

27. Ferreira, A.C.S.; Oliveira, C.; Hogg, T.; de Pinho, P.G. Relationship between potentiometric measurements, sensorial analysis, and some substances responsible for aroma degradation of white wines. J. Agric. Food Chem. 2003, 51, 4668-4672. [CrossRef] 